\title{
DICOM Header Tag
}

National Cancer Institute

\section{Source}

National Cancer Institute. DICOM Header Tag. NCI Thesaurus. Code C94884.

A uniquely defined quantity appearing in the computer file created according to the DICOM standard of transmitting images. It is important for the quantification of image features that many numerical values associated with the scanner configuration and protocol used be given. Each header tag is one such value. 